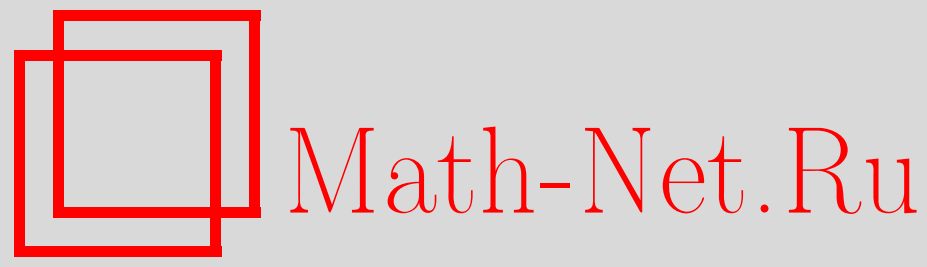

А. Г. Хованский, Теория пересечений и функция Гильберта, Функи. анализ и его прил., 2011, том 45, выпуск 4, 82-94

DOI: https://doi.org/10.4213/faa3044

Использование Общероссийского математического портала MathNet.Ru подразумевает, что вы прочитали и согласны с пользовательским соглашением

http://www . mathnet.ru/rus/agreement

Параметры загрузки:

IP : 34.239 .49 .27

26 апреля 2023 г., $17: 11: 43$






\title{
Теория пересечений и функция Гильберта*
}

\author{
(c) 2011. А. Г. ХовАнский
}

\section{Памяти Владимира Игоревича Арнольда}

\begin{abstract}
Бирационально-инвариантная теория пересечений является далеким обобщением и развитием теоремы Бернштейна-Кушниренко. В статье приводятся прозрачные доказательства теоремы Гильберта о степени проективного многообразия и ряда близких утверждений, играющих важную роль в этой теории. Статью можно читать независимо - в ней напоминаются все необходимые определения и результаты.
\end{abstract}

Введение. Теорема Гильберта (см. разд. 7) связывает степень проективного многообразия с асимптотикой его функции Гильберта. В статье даются элементарные доказательства этой теоремы над полем комплексных чисел и ряда близких утверждений, некоторые из которых (теорема 10) не очевидны с точки зрения алгебраической геометрии.

Зачем снова доказывать классическую теорему, входящую в учебники по алгебраической геометрии? Эта фундаментальная теорема играет ключевую роль для недавно появившейся и находящейся в процессе построения бирациональноинвариантной теории пересечений, как для ее комплексно-аналитической версии, так и версии, справедливой над любым алгебраически замкнутым полем. Для алгебраической версии нужна теорема Гильберта в ее стандартной форме. Для аналитической версии естественно иметь аналитическое доказательство. Оно дает новое видение предмета и подсказывает новые алгебраические результаты.

0.1. Бирационально-инвариантная теория пересечений. В 1975 г. была найдена знаменитая теорема Бернштейна-Кушниренко о числе решений в $\left(\mathbb{C}^{*}\right)^{n}$ общей системы из $n$ уравнений с заданными многогранниками Ньютона (доказательство, основанное на теореме Гильберта, можно найти в [1], [2]). Ее появлению способствовал богатый эмпирический материал, накопленный Владимиром Игоревичем Арнольдом при исследовании критических точек функций. Она послужила началом теории многогранников Ньютона, которая в рамках геометрии торических многообразий связала выпуклую геометрию с алгебраической геометрией и с теорией особенностей ([3], [4]). Эта теория интенсивно разрабатывалась (в частности, на семинаре Арнольда), ей посвящены сотни публикаций.

Теорема Бернштейна-Кушниренко не укладывается в рамки обычной теории пересечений: в ней идет речь о числе решений системы уравнений на неполном многообразии $\left(\mathbb{C}^{*}\right)^{n}$, левые части уравнений которой - достаточно общие функции из специальных конечномерных пространств. Ответ выражается в терминах многогранников Ньютона, само определение которых использует специфику этих пространств и многообразия $\left(\mathbb{C}^{*}\right)^{n}$.

\footnotetext{
*Работа выполнена при частичной поддержке Канадского гранта no. 0GP0156833.
} 
В бирационально-инвариантной теории пересечений вместо $\left(\mathbb{C}^{*}\right)^{n}$ берется любое (не обязательно полное) неприводимое $n$-мерное алгебраическое многообразие $X$ и рассматриваются системы, левые части уравнений которых - общие функции из произвольных конечномерных пространств рациональных функций на $X$. Эти конечномерные пространства образуют полугруппу (по умножению) $\mathcal{K}(X)$. Индекс пересечения $n$ элементов из $\mathcal{K}(X)$ - это (правильно интерпретированное) число решений системы, левые части уравнений которой - общие функции из этих $n$ пространств. Индекс пересечения автоматически переносится на группу Гротендика полугруппы $\mathcal{K}(X)$. Каждому пространству $L \in \mathcal{K}(X)$ сопоставляется его тело Нъютона-Окунъкова $\Delta(L) \subset \mathbb{R}^{n}$ таким образом, что $\Delta\left(L_{1}\right)+\Delta\left(L_{2}\right) \subset \Delta\left(L_{1} L_{2}\right)$ и что индекс самопересечения пространства $L$ равен (как в теореме Кушниренко) умноженному на $n$ ! объему тела $\Delta(L)$ (конструкция тела $\Delta(L)$ неоднозначна и содержит функциональные параметры, которые можно выбирать произвольно). Развиваемая теория связывает алгебру и геометрию вне рамок торической геометрии (см. [5], а также [6]).

Эта связь полезна в различных направлениях. Для алгебраической геометрии она доставляет элементарные доказательства аналогов геометрических неравенств Александрова-Фенхеля в теории пересечений ([5], [8]) и далекое обобщение теоремы об аппроксимации Фуджиты ([6], [8]). Для теории инвариантов она доставляет аналоги теоремы Бернштейна-Кушниренко для оросферических многообразий [9] и некоторых других многообразий, снабженных действием редуктивной группы ([10], [11]), и позволяет вычислить группу Гротендика полугруппы представлений редуктивной группы [10] (рассматриваемых с точностью до спектральной эквивалентности). В абстрактной алгебре она позволяет выделить широкий класс градуированных алгебр, функции Гильберта которых не совпадают при больших значениях аргумента с полиномом, но имеют полиномиальную асимптотику, а константы, характеризующие асимптотику, удовлетворяют аналогу геометрического неравенства Брунна-Минковского [8]. Для геометрии найденная связь доставляет прозрачное доказательство неравенства Александрова-Фенхеля и его многочисленных следствий ([5], [8]). Связь основана на геометрической теории полугрупп целых точек [8].

Опубликована часть комплексно-аналитической версии теории, содержащая ее глобальный вариант и вариант, связанный с действием редуктивных групп (см. список литературы). Мы с К. Каве записываем локальный вариант, доставляющий новые неравенства (аналогичные неравенству Александрова-Фенхеля) для кратностей примарных идеалов в локальном кольце. Соображения из настоящей статьи применяются для топологического доказательства формулы Самюэля для кратности корня системы уравнений в особой точке многообразия.

Мы записываем также версию теории, справедливую над любым алгебраически замкнутым полем (здесь мы пользуемся теоремой Гильберта в стандартной форме). Мы получаем абстрактные аналоги всех результатов, включая аналог для абстрактных локальных колец наших новых неравенств для кратностей примарных идеалов.

0.2. Содержание статьи. Пусть $X$ - неприводимое $n$-мерное комплексное алгебраическое многообразие и $\mathcal{K}(X)$ - полугруппа по умножению ненулевых конечномерных подпространств над $\mathbb{C}$ поля рациональных функций $\mathbb{C}(X)$ на $X$. Функиия Гильберта $H_{L}$ и нормализованная функиия Гильберта $\bar{H}_{L}$ пространства $L \in \mathcal{K}(X)$ определяются равенствами $H_{L}(k)=\operatorname{dim}_{\mathbb{C}} L^{k}$ и $\bar{H}_{L}(k)=$ 
$\operatorname{dim}_{\mathbb{C}} \overline{L^{k}}$, где $\overline{L^{k}}$ - целое замыкание пространства $L^{k}$ в поле $\mathbb{C}(X)$ (см. разд. 2 и 6). Асимптотики функций $H_{L} \leqslant \bar{H}_{L}$ связаны с индексом самопересечения $d$ пространства $L$.

Ситуация наиболее проста, если пространство $L$ разделяет общие точки в $X$. В этом случае существуют верхние и нижние оценки функций $H_{L} \leqslant \bar{H}_{L}$, имеющие одинаковые асимптотики при $k \rightarrow \infty$ и зависящие лишь от $n$ и $d$ (a не от $X$ и $L)$. Доказательства основаны на свойствах индексов пересечения на алгебраических многообразиях и на несложных рассуждениях, справедливых для любых аналитических многообразий.

Из оценок вытекает, что

$$
n ! \lim _{k \rightarrow \infty} \frac{H_{L}(k)}{n^{k}}=d \quad \text { и } n ! \lim _{k \rightarrow \infty} \frac{\bar{H}_{L}(k)}{n^{k}}=d .
$$

Первое из этих равенств эквивалентно теореме Гильберта для неприводимого проективного многообразия над полем $\mathbb{C}$ и дает самое простое из известных мне доказательств этой теоремы. Для приводимых проективных многообразий не существует двусторонних оценок функции Гильберта, зависящих лишь от $n$ и $d$ и имеющих правильные асимптотики.

Если пространство $L \in \mathcal{K}(X)$ не разделяет общие точки в $X$, то равенство $n ! \lim _{k \rightarrow \infty} H_{L}(k) / n^{k}=d$ не выполняется. Если $d>0$, то, во-первых, существует верхняя оценка функции $\bar{H}_{L}$, зависящая лишь от $n$ и $d$ и имеющая нужную асимптотику, и, во-вторых, справедливо равенство $d=n ! \lim \bar{H}_{L}(k) / n^{k}($ нижней оценки подобного вида для функции $\bar{H}_{L}$ не существует). В общем случае, без предположения $d>0$, не существует и верхней оценки подобного вида для функции $\bar{H}_{L}$, но равенство $d=n ! \lim \bar{H}_{L}(k) / n^{k}$ по-прежнему справедливо. Оно и доставляет связь асимптотики нормализованной функции Гильберта $\bar{H}_{L}$ и индекса самопересечения пространства $L$.

0.3. Расположение материала. Мы напоминаем нужные положения бирационально-инвариантной теории пересечений в разд. 1 и 2. В разд. 3 вводятся обозначения, используемые в разд. 4 и 5 при оценке размерностей некоторых пространств аналитических функций. В разд. 6 напоминаются свойства целого замыкания и дается простое доказательство того, что $\operatorname{dim}_{\mathbb{C}} \bar{L}<\infty$ при $L \in$ $\mathcal{K}(X)$. В разд. 7 разбирается случай, когда $L$ разделяет общие точки в $X$, в разд. 8 - случай, когда $d>0$. В разд. 9 в общем случае доказывается связь асимптотики функции $\bar{H}_{L}$ и индекса самопересечения.

1. Индекс пересечения в полугруппе $\mathcal{K}(\boldsymbol{X})$. Пусть $X-$ неприводимое $n$-мерное алгебраическое многообразие (в статье под алгебраическим многообразием мы подразумеваем квазипроективное алгебраическое многообразие) над полем $\mathbb{C}$. Пусть $\mathcal{K}(X)$ - множество, состоящее из ненулевых конечномерных подпространств над $\mathbb{C}$ поля $\mathbb{C}(X)$ рациональных функций на $X$. Множество $\mathcal{K}(X)$ наделено структурой коммутативной полугруппы относительно следующей операции умножения: произведением пространств $L, M \in \mathcal{K}(X)$ называется пространство $L M \in \mathcal{K}(X)$, натянутое на все функции вида $f g$, где $f \in L$, $g \in M$.

Для всякого набора $L_{1}, \ldots, L_{n} \in \mathcal{K}(X)$ определен (см. [7]) индекс пересечения $\left[L_{1}, \ldots, L_{n}\right]$ : число $\left[L_{1}, \ldots, L_{n}\right]$ равно числу решений в $X$ системы уравнений 
$f_{1}=\cdots=f_{n}=0$, где $f_{1} \in L_{1}, \ldots, f_{n} \in L_{n}$ - общий набор функций из пространств $L_{1}, \ldots, L_{n}$. При подсчете числа решений не учитываются решения, в которых обращаются в нуль все функции из некоторого пространства $L_{i}$, где $1 \leqslant i \leqslant n$, т. е. такие решения $a \in X$, что $f(a)=0$, если $f \in L_{i}$. Не учитываются также решения, в которых хотя бы одна функция $g$ из одного из пространств $L_{j}$ имеет полюс. Напомним основные свойства индекса пересечения (см. [7]).

1) Индекс пересечения определен корректно. Скажем, что некоторым свойством обладает общий элемент линейного пространства $M$ над $\mathbb{C}$, если существует комплексное полуалгебраическое множество $\Sigma \subset M$, такое, что этим свойством обладают элементы множества $M \backslash \Sigma$ и $\operatorname{dim} \Sigma<\operatorname{dim} M$. Пусть $L_{1}, \ldots, L_{n} \in \mathcal{K}(X)$ - любой набор пространств и $O \subset X-$ любое комплексное полуалгебраическое подмножество, такое, что

(a) $O$ содержит все особые точки многообразия $X$,

(b) для $1 \leqslant i \leqslant n$ справедливо включение $O_{i} \subset O$, где $O_{i}-$ множество точек, в которых обращаются в нуль все функции из пространства $L_{i}$,

(c) $O$ содержит объединение дивизоров полюсов всех функций из пространств $L_{1}, \ldots, L_{n}$,

(d) выполнено неравенство $\operatorname{dim} O<\operatorname{dim} X$.

Под корректностью определения индекса пересечения мы понимаем следующее утверждение: для общего элемента $\left(f_{1}, \ldots, f_{n}\right) \in L_{1} \times \cdots \times L_{n}$ все корни системы уравнений $f_{1}=\cdots=f_{n}=0$, лежащие в множестве $X \backslash O$, невырожденны (т.е. дифференииаль $d f_{i}$ линейно независимы в каждом корне) и их число не зависит от выбора множества $O$, удовлетворяющего условиям (а) $-(d)$, и равно $\left[L_{1}, \ldots, L_{n}\right]$.

2) Индекс пересечения симметричен, т. е. не меняется при перестановке пространств. Это свойство непосредственно вытекает из определения.

3) Индекс пересечения полилинеен. Линейность индекса по первому аргументу означает, что для любых пространств $L_{1}^{\prime}, L_{1}^{\prime \prime}, L_{2}, \ldots, L_{n} \in \mathcal{K}(X)$ справедливо равенство $\left[L_{1}^{\prime} L_{1}^{\prime \prime}, L_{2}, \ldots, L_{n}\right]=\left[L_{1}^{\prime}, L_{2}, \ldots, L_{n}\right]+\left[L_{1}^{\prime \prime}, L_{2}, \ldots, L_{n}\right]$. Линейность по остальным аргументам определяется аналогично.

2. Группа Гротендика полугруппы $\mathcal{K}(\boldsymbol{X})$. Как и для всякой коммутативной полугруппы, для полугруппы $\mathcal{K}(X)$ определены ее полугруппа Гротендика и ее группа Гротендика.

Определение 1. Два элемента $a, b$ коммутативной полугруппы $S$ называются эквивалентными, $a \sim b$, если существует элемент $c \in S$, такой, что $a c=b c$. Множество классов эквивалентности с индуцированной операцией умножения образует коммутативную полугруппу с сокращением (т. е. полугруппу, в которой из равенства $A C=B C$ вытекает, что $A=B)$, называемую полугруппой Гротендика полугруппы $S$. Полугруппу Гротендика полугруппы $\mathcal{K}(X)$ мы будем обозначать через $\mathcal{K}_{G}(X)$.

Определение 2. С коммутативной полугруппой $S$ связано множество $G(S)$ пар $(a, b)$ элементов $a, b$ ее полугруппы Гротендика, в котором введено отождествление $(a, b) \sim(c, d) \Longleftrightarrow a d=b c$. Множество $G(S)$ с операцией умножения $(a, b)(c, d)=(a c, b d)$ и операцией обращения $(a, b)^{-1}=(b, a)$ является группой, которая называется группой Гротендика полугруппы $S$. Группу Гротендика полугруппы $\mathcal{K}(X)$ будем обозначать через $G(X)$.

Всякий гомоморфизм $\tau: S \rightarrow G$ коммутативной полугруппы $S$ в коммутативную группу $G$ однозначно пропускается через естественное отображение 
$\rho: S \rightarrow G(S)$ полугруппы $S$ в ее группу Гротендика, т. е. существует единственный гомоморфизм $\tilde{\tau}: G(S) \rightarrow G$, такой, что $\tau=\tilde{\tau} \circ \rho$.

Утверждение 1. Пустъ $L_{1}, \ldots, L_{n} \in \mathcal{K}(X)$ u $L_{1}^{\prime}, \ldots, L_{n}^{\prime} \in \mathcal{K}(X)$, причем $L_{1} \sim L_{1}^{\prime}, \ldots, L_{n} \sim L_{n}^{\prime}$. Тогда $\left[L_{1}, \ldots, L_{n}\right]=\left[L_{1}^{\prime}, \ldots, L_{n}^{\prime}\right]$.

Доказательство. Покажем, что $\left[L_{1}, L_{2}, \ldots, L_{n}\right]=\left[L_{1}^{\prime}, L_{2}, \ldots, L_{n}\right]$. Действительно, по линейности индекса пересечения отображение $\mathcal{K}(X) \mapsto \mathbb{Z}$, сопоставляющее элементу $L \in \mathcal{K}(X)$ число $\left[L, L_{2}, \ldots, L_{n}\right] \in \mathbb{Z}$, является гомоморфизмом полугруппы $\mathcal{K}(X)$ в группу $\mathbb{Z}$. Этот гомоморфизм продолжается на группу Гротендика, откуда и вытекает нужное равенство. В силу симметрии индекса пересечения все остальные пространства тоже можно заменить на эквивалентные, не меняя индекса пересечения.

Обсудим очевидные свойства соотношения эквивалентности $L_{1} \sim L_{2}$ в полугруппе $\mathcal{K}(X)$ :

Утверждение 2. (i) Если $L_{1} \sim L_{2}$, то $L_{1}+L_{2} \sim L_{1} \sim L_{2}$.

(ii) Если $L_{1} \sim L_{2}$ и выполняются включения $L_{1} \subset L \subset L_{2}$, то $L_{1} \sim L \sim L_{2}$.

Доказательство. (i) Если $L_{1} M=L_{2} M$, то $\left(L_{1}+L_{2}\right) M=L_{1} M+L_{2} M=$ $L_{1} M=L_{2} M$.

(ii) Если $L_{1} \subset L \subset L_{2}$ и $L_{1} M=L_{2} M$, то $L_{1} M \subset L M \subset L_{2} M$. Поэтому $L_{1} M=L M=L_{2} M$.

Скажем, что функция $f \in \mathbb{C}(X)$ тривиальна над $L \in \mathcal{K}(X)$, если $L \sim L(f)$, где $L(f)$ - пространство, порожденное функциями из $L$ и функцией $f$.

Утверждение 3. (i) Если $L \subset M, L \sim M u f \in M$, mo $L(f) \sim L$.

(ii) Тривиальные над $L \in \mathcal{K}(X)$ функиии образуют линейное пространство над $\mathbb{C}$.

(iii) Если $L(f) \sim L$ и g тривиальна над $L(f)$, то $L(g) \sim L$.

(iv) Если $f$ тривиальна над $L^{k}$ и $g$ тривиальна над $L^{m}$, то $f g$ тривиальна над $L^{k+m}$.

Доказательство. (i) Имеем $L \subset L(f) \subset M$. Так как $L \sim M$, то $L(f) \sim L$.

(ii) Пусть $L(f) \sim L$ и $L(g) \sim L$. Тогда $L(f)+L(g) \sim L$. Для $\lambda, \mu \in \mathbb{C}$ имеем $L \subset L(\lambda f+\mu g) \subset L(f)+L(g)$. Поэтому $L(\lambda f+\mu g) \sim L$.

(iii) Положим $L^{\prime}=L(f)$. Имеем $L^{\prime} \sim L$ и $L^{\prime}(g) \sim L^{\prime}$, откуда $L^{\prime}(g) \sim L$ и, следовательно, $L(g) \sim L$.

(iv) Если $L^{k}(f) \sim L^{k}$ и $L^{m}(g) \sim L^{m}$, то $L^{k}(f) L^{m}(g) \sim L^{k+m}$. Далее $L^{k+m} \subset$ $L^{k}(f) L^{m}(g)$ и $f g \in L^{k}(f) L^{m}(g)$, поэтому $L^{k+m}(f g) \sim L^{k+m}$.

Тривиальность функции $f$ над пространством $L$ можно описать совершенно в других терминах (см. [7], [12]).

Определение 3. Функция $f \in \mathbb{C}(X)$ называется целой функцией над $L \in$ $\mathcal{K}(X)$, если она удовлетворяет некоторому уравнению

$$
f^{d}+a_{1} f^{d-1}+\cdots+a_{d}=0,
$$

коэффициенты $a_{i}$ которого лежат в пространствах $L^{i}$.

Утверждение 4. $L(f) \sim L$, если и толъко если $f$ - целая функиия над $L$. Доказательство. Если $f$ удовлетворяет уравнению $(1)$, то $L(f)(L(f))^{d}=$ $L(L(f))^{d}$. Это доказывает, что целая функция над $L$ тривиальна над $L$. 
Пусть $L(f) \sim L$. Тогда есть $M \in \mathcal{K}(X)$, такое, что $L M=L(f) M$. Пусть $e_{1}, \ldots, e_{d}$ - базис пространства $M$ над полем $\mathbb{C}$. Равенство $L M=L(f) M$ означает, что выполняются тождества $f e_{i}=\sum b_{i, j} e_{j}$, где $b_{i, j}$ - некоторые элементы пространства $L$. Поэтому $f$ является корнем характеристического полинома $\operatorname{det}(B-f I)=0 \quad(d \times d)$-матрицы $B=\left\{b_{i, j}\right\}$. Это доказывает, что если $L(f) \sim L$, то $f$ - целая функция над $L$.

Следствие 5. Пусть $L(f) \sim L$ и все функиии из $L \in \mathcal{K}(X)$ регулярны в области $U \subset X$. Тогда $f$ регулярна в $U$.

3. Обозначения. Ниже, в пп. 3.1 и 3.2 , определяются специальные целочисленные функции, в терминах которых формулируются оценки из разд. 4 и 5 . В п. 3.3 вводятся общие для этих пунктов обозначения.

3.1. Пусть $Q(n, l)$ - размерность пространства полиномов степени $\leqslant l$ от $n$ переменных. Число $Q(n, l)$ равно числу целых точек в $n$-мерном симплексе $\Delta$, заданном неравенствами $0 \leqslant u_{1}, \ldots, 0 \leqslant u_{n}, u_{1}+\cdots+u_{n} \leqslant l$. Объем симплекса $\Delta$ равен $l^{n} / n$ !. Поэтому при заданном $n$ и $l \rightarrow \infty$ имеем $Q(n, l) \approx l^{n} / n !$. Легко видеть, что $Q(n, l)=C_{l+n}^{n}$.

Пространство однородных полиномов степени $k$ от $n+1$ переменных изоморфно пространству полиномов степени $\leqslant k$ от $n$ переменных. Поэтому $Q(n+1, k)-Q(n+1, k-1)=Q(n, k)$. Отсюда получаем равенство

$$
Q(n+1, k)-Q(n+1, k-d)=Q(n, k)+Q(n, k-1)+\cdots+Q(n, k-d+1) .
$$

3.2. В разд. 5 нам понадобится следующее определение.

Определение 4. Пусть $N=k d+r$, где $r, 0 \leqslant r<d$, - остаток от деления числа $N$ на число $d$. Определим функцию $F$ от $(n, d, N)$ формулой

$$
F(n, d, N)=r Q(n, k)+(d-r) Q(n, k-1) .
$$

При заданных $n, d$ и $N \rightarrow \infty$ имеем $F(n, d, N) \approx d k^{n} / n !$, где $k=[N / d]$.

3.3. В разд. 4 и 5 мы будем использовать следующие обозначения:

$X^{*}-n$-мерное комплексно-аналитическое многообразие;

$L$ - конечномерное пространство аналитических функций на $X^{*}$, содержащее константы; мы будем предполагать, что $L$ содержит набор функций $x_{1}, \ldots, x_{n}$, такой, что в множестве решений системы уравнений $x_{1}=\cdots=$ $x_{n}=0$ на $X^{*}$ найдется подмножество $Y=\left\{y_{1}, \ldots, y_{d}\right\}$, состоящее из невырожденных решений (т. е. в точках множества $Y$ дифференциалы $d x_{i}$ функций $x_{i}$ для $i=1, \ldots, n$ линейно независимы);

$\mathbf{x}: X^{*} \rightarrow \mathbb{C}^{n}$ - отображение, заданное формулой $\mathbf{x}(q)=\left(x_{1}(q), \ldots, x_{n}(q)\right)$;

$\mathbf{x}_{i}^{-1}: U \rightarrow V_{i}$ - локальное обращение отображения $\mathbf{x}$, такое, что $\mathbf{x}_{i}^{-1}(0)=y_{i}$, $V_{i}$ - окрестность точки $y_{i}$ и $U$ - окрестность точки 0 (общая для всех $i$, $1 \leqslant i \leqslant d)$.

4. Оценка размерности снизу. В следующей простой лемме 6 мы пользуемся обозначениями, введенными в разд. 3.

Лемма 6. 1. Пусть функиия $f \in L$ принимает в точках $y_{1}, \ldots, y_{d}$ различные значения. Тогда $f$ не удовлетворяет никакому уравнению

$$
a_{1}(\mathbf{x}) f^{d-1}+\cdots+a_{d}(\mathbf{x})=0,
$$

в котором $a_{1}, \ldots, a_{d}-$ полиномы на $\mathbb{C}^{n}$ (не равные нулю одновременно). 
2. Пусть существует функиия $f \in L$, принимающая в точках $y_{1}, \ldots, y_{d}$ различные значения. Тогда

$\operatorname{dim}_{\mathbb{C}} L^{k} \geqslant Q(n+1, k)-Q(n+1, k-d)=Q(n, k)+Q(n, k-1)+\cdots+Q(n, k-d+1)$.

Доказательство. 1. Пусть $f_{i}$ - функция в области $U$, заданная формулой $f_{i}=f\left(\mathbf{x}_{i}^{-1}\right)$. При разных $i, j$ функции $f_{i}, f_{j}$ в окрестности $U$ не совпадают и удовлетворяют уравнению $a_{1} y^{d-1}+\cdots+a_{d}=0$. Но уравнение степени $d-1$ имеет не более $d-1$ корней. Противоречие доказывает п. 1.

2. Пространство $L^{k}$ содержит функции $a_{1}(\mathbf{x}) f^{d-1}+\cdots+a_{d}(\mathbf{x})$, в которых $a_{i}$ - полиномы от $x_{1}, \ldots, x_{n}$ степени, меньшей или равной $k-d+i$. По п. 1 эти функции линейно независимы. Лемма 6 доказана.

Скажем, что пространство $L \in \mathcal{K}(X)$ разделяет общие точки в алгебраическом многообразии $X$, если существует полуалгебраическое множество $O(L) \subset$ $X$, такое, что: (i) $\operatorname{dim} O(L)<\operatorname{dim} X$; (ii) $O(L)$ содержит дивизор полюсов функций из $L$; (iii) для любых различных точек $a, b \in X \backslash O(L)$ существует функция $g \in L$, такая, что $g(a) \neq g(b)$.

Лемма 7. Пусть $L \in \mathcal{K}(X)$ разделяет общие точки в $X$ и $Y \subset X$ - конечное множество, не пересекающееся с $O(L)$. Тогда существует функиия $f \in L$, принимающая разные значения в различных точках множества $Y$.

Доказательство. Множество функций из $L$, принимающих равные значения в паре различных точек из $Y$, является собственным подпространством в $L$. Объединение конечного числа собственных подпространств не может совпадать c $L$.

Теорема 8. Пусть $L \in \mathcal{K}(X)$ разделяет общие точки на неприводимом $n$-мерном алгебрачческом многообразии $X u[L, \ldots, L]=d>0$. Тогда $\operatorname{dim}_{\mathbb{C}} L^{k} \geqslant Q(n+1, k)-Q(n+1, k-d)=Q(n, k)+Q(n, k-1)+\cdots+Q(n, k-d+1)$.

Доказательство. Положим $X^{*}=X \backslash(D \cup O)$, где $D$ - дивизор полюсов функций из пространства $L$ и $O$ - объединение множества особых точек многообразия $X$ с множеством точек, на которых обращаются в нуль все функции из пространства $L$. По лемме 7 найдется функция $f \in L$, принимающая в точках $y_{1}, \ldots, y_{d}$ различные значения. Для завершения доказательства осталось воспользоваться п. 2 леммы 6.

5. Оценка размерности сверху. В формулировке леммы 9 мы используем обозначения из разд. 3 и предполагаем, что многообразие $X^{*}$ связно.

Лемма 9. Пусть $M$ - линейное пространство аналитических функций на $X^{*}$, содержащее константы и такое, что $\operatorname{dim}_{\mathbb{C}} M>F(d, n, N)$. Тогда есть функиии $l_{1}, \ldots, l_{n-1} \in L$ u $\varphi \in M$, такие, что система уравнений $l_{1}=\cdots=$ $l_{n-1}=\varphi=0$ имеет не меньше чем $N$ невырожденных решений в $X^{*}$.

Доказательство. Выделим $r$ точек $y_{1}, \ldots, y_{r}$ из множества $Y$. Пусть $\Omega_{k, Y}-$ линейное пространство, точка которого - это набор $k$-струй гладких функций в точках $y_{1}, \ldots, y_{r}$ и набор $(k-1)$-струй гладких функций в точках $y_{r+1}, \ldots, y_{d}$. Ясно, что $\operatorname{dim}_{\mathbb{C}} \Omega_{k, Y}=F(n, d, N)<\operatorname{dim}_{\mathbb{C}} M$. Поэтому существует ненулевая функция $g \in M$, которая переходит в нуль при отображении $\tau: M \rightarrow \Omega_{k, Y}$, сопоставляющем функции набор ее $k$-струй в точках $y_{1}, \ldots, y_{r}$ и набор ее $(k-1)$-струй в точках $y_{r+1}, \ldots, y_{d}$. 
Если $h$ - однородная линейная функция на $\mathbb{C}^{n}$, то функция $h(\mathbf{x})$ лежит в $L$. Рассмотрим однородную систему линейных уравнений $h_{1}=\cdots=h_{n-1}=0$ в $\mathbb{C}^{n}$. Ей соответствует система уравнений $l_{1}=\cdots=l_{n-1}=0$ в $X^{*}$, где $l_{i}=h_{i}(\mathbf{x})$, которая определяет гладкие кривые $\Gamma_{1}, \ldots, \Gamma_{d}$ в областях $V_{1}, \ldots, V_{d}$, проходящие через точки $y_{1}, \ldots, y_{d}$.

Если уравнения $h_{1}=\cdots=h_{n-1}=0$ достаточно общие, то ограничения функции $g$ на кривые $\Gamma_{1}, \ldots, \Gamma_{d}$ не равны тождественно нулю и имеют нули кратностей $\geqslant k+1$ в точках $y_{1}, \ldots, y_{r}$ и нули кратности $\geqslant k$ в точках $y_{r+1}, \ldots, y_{d}$. Действительно, отображение х отождествляет окрестности $V_{i} \subset X^{*}$ точек $y_{i}$ с окрестностью $U \subset \mathbb{C}^{n}$ точки 0. При этом отождествлении каждая из кривых $\Gamma_{1}, \ldots, \Gamma_{d}$ переходит в часть прямой $h_{1}=\cdots=h_{n-1}=0$, лежащую в области $U$. Ограничение функции $g$ на область $V_{i}$ переходит в функцию $g_{i}=g\left(\mathbf{x}_{i}^{-1}\right)$ на области $U$, не равную тождественному нулю (в противном случае в силу аналитичности функции $g$ и связности многообразия $X^{*}$ функция $g$ тоже тождественно равна нулю, что не так). Раз функции $g_{1}, \ldots, g_{d}$ не равны тождественному нулю в области $U$, то для почти всякой прямой $h_{1}=\cdots=h_{n-1}=0$ ограничение каждой из функций $g_{i}$ на эту прямую не обращается в тождественный нуль.

Поэтому система уравнений $l_{1}=\cdots=l_{n-1}=\varphi=0$, где $\varphi=g-\varepsilon=0$, при малом $\varepsilon$ будет иметь не меньше чем $N$ невырожденных корней: не меньше чем по $k+1$ невырожденных корней на кривых $\Gamma_{1}, \ldots, \Gamma_{r}$ и не меньше чем по $k$ невырожденных корней на кривых $\Gamma_{r+1}, \ldots, \Gamma_{d}$. Лемма 9 доказана, так как $(k+1) r+k(d-r)=k d+r=N$.

Теорема 10. Пусть $X$ - неприводимое $n$-мерное алгебраическое многообразие, $L \in \mathcal{K}(X) u[L, \ldots, L]=d>0$. Тогда если $M \in \mathcal{K}(X) u[L, \ldots, L, M] \leqslant N$, $m o \operatorname{dim}_{\mathbb{C}} M \leqslant F(n, d, N+1)$.

Доказательство. Положим $X^{*}=X \backslash(D \cup O)$, где $D$ - дивизор полюсов функций из пространств $L$ и $M$ и $O$ - объединение множества особых точек многообразия $X$ с множествами точек $O_{1}$ и $O_{2}$, на которых обращаются в нуль соответственно все функции из пространств $L$ и $M$. Если $\operatorname{dim} M>F(n, d, N+1)$, то по лемме 9 найдутся функции $l_{1}, \ldots, l_{n-1} \in L$ и $\phi \in M$, такие, что система $l_{1}=\cdots=l_{n-1}=\phi=0$ имеет не менее чем $N+1$ невырожденных корней в $X^{*}$, что противоречит неравенству $[L, \ldots, L, M] \leqslant N$. Теорема 10 доказана.

Следствие 11. В условиях теоремы 10

(i) если $M \sim L$, mo $\operatorname{dim}_{\mathbb{C}} M \leqslant n+d$;

(ii) ecлu $M \sim L^{k}$, mo $\operatorname{dim}_{\mathbb{C}} M \leqslant Q(n, k+1)+(d-1) Q(n, k)$.

Доказательство. Пункт (ii) вытекает из теоремы 10 , так как $\left[L, \ldots, L, L^{k}\right]=$ $k d$ и $F(n, d, k d+1)=Q(n, k)+(d-1) Q(n, k-1)$. Пункт (i) вытекает из п. (ii), так как $Q(n, 1)=n+1$ и $Q(n, 0)=1$.

6. Целое замыкание подпространства. Вернемся к эквивалентности, превращающей полугруппу $\mathcal{K}(X)$ в ее полугруппу Гротендика $\mathcal{K}_{G}(X)$.

Определение 5. Для всякого пространства $L \in \mathcal{K}(X)$ определим его целое замыкание $\bar{L}$ как множество всех функций $f \in \mathbb{C}(X)$, целых над $L$.

Из утверждений 3 и 4 видно, что множество $\bar{L}$ является линейным пространством. Если $L, M \in \mathcal{K}(X)$ и $L \subset M$, то $\bar{L} \subset \bar{M}$.

Утверждение 12. Пусть $X$ - неприводимое $n$-мерное алгебраическое многообразие, $L \in \mathcal{K}(X) u[L, \ldots, L]=d>0$. Тогда $\operatorname{dim}_{\mathbb{C}} \bar{L} \leqslant d+n$. 
Доказательство вытекает из следствия 11, п. (ii).

Следствие 13. (i) Если $L \in \mathcal{K}(X)$, то $\bar{L}$ имеет конечную размерность, m.e. $\bar{L} \in \mathcal{K}(X)$.

(ii) Для $M \in \mathcal{K}(X)$ имеем $M \sim L \Longleftrightarrow \bar{M}=\bar{L}$.

Доказательство. Пункт (i) в случае $[L, \ldots, L]>0$ доказан в утверждении 12 . Если $[L, \ldots, L]=0$, то вместо $L$ возьмем любое большее пространство $L \subset M$, такое, что $[M, \ldots, M]>0$. Согласно утверждению $12, \operatorname{dim}_{\mathbb{C}} \bar{M}<\infty$. Пункт (i) доказан, так как $\bar{L} \subset \bar{M}$.

(ii) Согласно п. (i), среди всех пространств, эквивалентных заданному пространству $L$, есть наибольшее пространство $\bar{L}$. Отсюда вытекает п. (ii).

Следствие 13 вытекает также из теоремы Нётер о целом замыкании ([7], [12]). Но наши оценки и здесь позволяют избежать ссылок на алгебраическую геометрию. Пример 1 показывает, что оценка из утверждения 12 неулучшаема.

Пример 1. Пусть $X=\mathbb{C}^{n}, x_{1}, \ldots, x_{n}-$ координаты в $\mathbb{C}^{n}$. Возьмем в качестве $L$ пространство, порожденное функциями $1, x_{1}, \ldots, x_{1}^{d}$ и функциями $x_{2}, \ldots, x_{n}$. Тогда $[L, \ldots, L]=d$ и $\operatorname{dim}_{\mathbb{C}} L=d+n$.

Определение 6. Для пространства $L \in \mathcal{K}(X)$ функиия Гилъберта $H_{L}$ и нормализованная функиия Гильберта $\bar{H}_{L}$ определяются равенствами

$$
H_{L}(k)=\operatorname{dim}_{\mathbb{C}} L^{k}, \quad \bar{H}_{L}(k)=\operatorname{dim}_{\mathbb{C}} \overline{L^{k}} .
$$

\section{7. Случай, когда пространство функций разделяет точки.}

Теорема 14. Пусть $L \in \mathcal{K}(X)$ разделяет общие точки неприводимого $n$-мерного алгебрачческого многообразия $X$. Пусть $[L, \ldots, L]=d>0$. Тогда

$$
\begin{gathered}
\sum_{k-d<i \leqslant k} Q(n, i) \leqslant H_{L}(k) \leqslant \bar{H}_{L}(k) \leqslant F(n, d, k d+1), \\
{[L, \ldots, L]=\lim _{k \rightarrow \infty} \frac{n ! H_{L}(k)}{k^{n}}=\lim _{k \rightarrow \infty} \frac{n ! \bar{H}_{L}(k)}{k^{n}} .}
\end{gathered}
$$

Доказательство. Соотношение (2) вытекает из теорем 8 и 10. Имеем $\sum_{k-d<i \leqslant k} Q(n, i) \approx F(n, d, k d+1) \approx d k^{n} / n$ !. Поэтому (3) вытекает из (2).

Пример 2. Оценка снизу $H_{L}(k) \geqslant \sum_{k-d<i \leqslant k} Q(n, i)$ точна для алгебраической гиперповерхности $X \subset \mathbb{C}^{n+1}$ степени $d$ и пространства $L$, порожденного координатными функциями в $\mathbb{C}^{n+1}$ и константами.

Пример 3. Пусть $X$ - неприводимая плоская алгебраическая кривая степени $d$, а $L-$ пространство, порожденное константами и координатными функциями. В этом случае нижняя оценка размерности $\operatorname{dim}_{\mathbb{C}} L^{k}$ точна (см. пример 2). Верхняя оценка $\bar{H}_{L}(k) \leqslant k d+1$ для кривой $X$ рода нуль точна, т. е. $\bar{H}_{L}(k)=k d+1$. При $k \gg 0$ для любого $m$, такого, что $1-(d-1)(d-2) / 2 \leqslant m \leqslant 1$, можно построить плоскую кривую, для которой нижняя оценка точна, а верхняя оценка есть $\operatorname{dim}_{\mathbb{C}} \overline{L^{k}}=k d+m$.

Пример 4. Если $[L, \ldots, L]=1$ и если $[L, \ldots, L]=2$, то верхняя оценка функции $\bar{H}_{L}(k)$ совпадает с нижней оценкой функции $H_{L}(k)$. В этом случае теорема дает формулу для функций $\bar{H}_{L}(k)=H_{L}(k)$. 
$C$ тепенъю $d(X)$ проективного $n$-мерного многообразия $X \subset \mathbb{C} P^{N}$ называется число точек его пересечения с общим проективным подпространством коразмерности $n$. С многообразием $X \subset \mathbb{C} P^{N}$ связан идеал $I_{X} \subset \mathbb{C}\left[x_{0}, \ldots, x_{N}\right]$, состоящий из полиномов от однородных координат $\left(x_{0}: \cdots: x_{N}\right)$ на $\mathbb{C} P^{N}$, обращающихся в нуль на $X$, и кольцо $A_{X}=\mathbb{C}\left[x_{0}, \ldots, x_{N}\right] / I_{X}$. Кольцо $A_{X}$ разлагается в прямую сумму однородных компонент $A_{X}=A_{0}+A_{1}+\ldots$ Функиией Гильберта $H_{[X]}$ многообразия $X \subset \mathbb{C} P^{N}$ называется функция на неотрицательных целых числах, определенная формулой $H_{[X]}(k)=\operatorname{dim}_{\mathbb{C}} A_{k}$.

Теорема Гильберта. Для любого неприводимого $n$-мерного проективного многообразия $X \subset \mathbb{C} P^{N}$ существует предел $l(X)=\lim _{k \rightarrow \infty} H_{[X]}(k) / k^{n}$. Более того, степень $d(X)$ многообразия $X$ равна $n ! l(X)$.

Замечание. В формулировку теоремы Гильберта обычно добавляется утверждение о полиномиальности функции $H_{X}$ при достаточно больших значениях аргумента. Это свойство функций Гильберта справедливо для любых конечно порожденных градуированных модулей над кольцом полиномов и не связано со степенью проективного многообразия. В теореме Гильберта можно не предполагать, что многообразие $X$ неприводимо (общий случай сводится к случаю неприводимого многообразия).

Доказательство теоремы Гильберта. Теорема 14 не только содержит теорему Гильберта, но и доставляет явные оценки функции Гильберта для любого значения аргумента. Действительно, пусть $D$ - общее гиперплоское сечение. Представим $\mathbb{C} P^{N}$ в виде объединения пространства $\mathbb{C}^{N}$ и «бесконечно удаленного» пространства $\mathbb{C} P^{N-1}$ и будем считать, что $D-$ пересечение многообразия $X$ с $\mathbb{C} P^{N-1}$.

Рассмотрим аффинное многообразие $X_{\mathrm{af}}=X \backslash D \subset \mathbb{C}^{N}$. На нем есть выделенное конечномерное пространство функций $L$, состоящее из ограничений на $X_{\text {af }}$ полиномов первой степени. Непосредственно из определения видно, что $H_{L}=H_{[X]}$. Степень $d(X)$ многообразия $X$ равна числу решений общей системы линейных уравнений $l_{1}=\cdots=l_{n}=0$ на $X_{\mathrm{af}}$, т. е. $d(X)=[L, \ldots, L]$.

Многообразие $X_{\mathrm{af}}$ с пространством функций $L$ удовлетворяют всем условиям теоремы 14. Действительно, $X_{\mathrm{af}} \subset \mathbb{C}^{N}$ и $L$ содержит ограничения на $X_{\text {af }}$ всех координатных функции на $\mathbb{C}^{N}$. Поэтому пространство $L$ разделяет точки на $X_{\text {af }}$. Покажем, что $[L, \ldots, L]>0$. Возьмем любое аффинное пространство $M$ коразмерности $n$, проходящее через какую-либо гладкую точку $a \in X_{\mathrm{af}}$ трансверсально к $X_{\mathrm{af}}$. Пространство $M$ задается системой уравнений $l_{1}=\cdots=l_{n}=0$, в которой $l_{i}$ - полиномы первой степени. Эта система на $X_{\mathrm{af}}$ имеет невырожденный корень $a$. Общая система уравнений $l_{1}=\cdots=l_{n}=0$ на $X_{\mathrm{af}}$, где $l_{i} \in L$, имеет не меньше невырожденных корней на $X_{\mathrm{af}}$. Поэтому $[L, \ldots, L]>0$. Теперь двусторонние оценки функции $H_{[X]}$ и теорема Гильберта вытекают из теоремы 14.

\section{8. Случай, когда самопересечение положительно.}

Утверждение 15. Пусть $X$ - неприводимое $n$-мерное алгебраическое многообразие, $L \in \mathcal{K}(X) u[L, \ldots, L]=d>0$. Рассмотрим отображение $\mathbf{x}: X \rightarrow$ $\mathbb{C}^{n}$, в котором $\mathbf{x}=\left(x_{1}, \ldots, x_{n}\right)$ - общий набор функиий из пространства $L$. Тогда всякая функиия $f \in \mathbb{C}(X)$ удовлетворяет уравнению $f^{d}+a_{1}(\mathbf{x}) f^{d-1}+\cdots+$ $a_{0}(\mathbf{x})=0$, в котором $a_{1}, \ldots, a_{d}$ - некоторье рациональнье функиии на $\mathbb{C}^{n}$. 
Доказательство. Так как $[L, \ldots, L]=d$, то в $\mathbb{C}^{n}$ существует полуалгебраическое множество $\Sigma \subset \mathbb{C}^{n}$, такое, что $\operatorname{dim} \Sigma<n$ и каждая точка $z \in \mathbb{C}^{n} \backslash \Sigma$ имеет ровно $d$ невырожденных прообразов $y_{i}=\mathbf{x}_{i}^{-1}(z)$ при отображении $\mathbf{x}: X \rightarrow \mathbb{C}^{n}$. Для $\phi \in \mathbb{C}(X)$ функция Trace $\mathbf{x} \phi$ на области $\mathbb{C}^{n} \backslash \Sigma$, определенная формулой Trace $_{\mathbf{x}} \phi=\sum_{i} \phi\left(\mathbf{x}_{i}^{-1}\right)$, продолжается до рациональной функции на $\mathbb{C}^{n}$. Это продолжение мы будем обозначать тем же символом $\operatorname{Trace}_{\mathbf{x}} \phi$. Для $k=0, \ldots, d-1$ рассмотрим симметрические функции Ньютона $N_{k}=\sum_{i} f^{k}\left(\mathbf{x}_{i}^{-1}\right)=$ Trace $_{\mathbf{x}} f^{k}$ от ветвей $f\left(\mathbf{x}_{i}^{-1}\right)$ многозначной функции $f\left(\mathbf{x}^{-1}\right)$. Пусть $S_{k}=P_{k}\left(N_{1}, \ldots, N_{k}\right)$ выражение базисной симметрической функции от ветвей $f\left(\mathbf{x}_{i}^{-1}\right)$ через симметрические функции Ньютона этих ветвей. Тогда $f^{d}-S_{1} f^{d-1}+\cdots+(-1)^{d} S_{d}=0$.

Следствие 16. В условиях утверждения 15 существует число $m$, такое, что пространство $\overline{L^{m}}$ содержит функиию $g$, разделяющую точки $y_{1}, \ldots, y_{d}$.

Доказательство. Пусть $z$ - точка области $\mathbb{C}^{n} \backslash \Sigma$ и $f \in \mathbb{C}(X)-$ функция, регулярная в точках множества $Y=(\mathbf{x})^{-1}(z)$ и принимающая разные значения в различных точках этого множества. Функция $f$ удовлетворяет уравнению $f^{d}-$ $S_{1} f^{d-1}+\cdots+(-1)^{d} S_{d}=0$, в котором $S_{i}-$ рациональные функции, регулярные в точке $z$. Функция $g=f Q$, где $Q$ - наименьшее общее кратное знаменателей рациональных функций $S_{1}, \ldots, S_{d}$, удовлетворяет уравнению $g^{d}-Q S_{1} g^{d-1}+$ $\cdots+(-1)^{d} Q^{d} S_{d}=0$ с полиномиальными коэффициентами $(-1)^{i} Q^{i} S_{i}$. Функция $g$ разделяет точки множества $Y$, так как $Q(z) \neq 0$, и функция $f$ разделяет точки множества $Y$. Осталось отметить, что функция $g$ лежит в пространстве $\overline{L^{m}}$, где $m$ - любое число, большее всех чисел $\operatorname{deg}\left(Q^{i} S_{i}\right) / i$ при $i=1, \ldots, d$.

Теорема 17. Пусть $X$ - неприводимое $n$-мерное алгебраическое многообразие, $L \in \mathcal{K}(X)$ u $[L, \ldots, L]=d>0$. Тогда

$$
\begin{gathered}
\bar{H}_{L}(k) \leqslant F(n, d, k d+1), \\
{[L, \ldots, L]=\lim _{k \rightarrow \infty} \frac{n ! \bar{H}_{L}(k)}{k^{n}} .}
\end{gathered}
$$

Доказательство. Неравенство (4) вытекает из теоремы 10. По следствию 16 при некотором $m$ пространство $\overline{L^{m}}$ разделяет общие точки в $X$. Имеем $\left[\overline{L^{m}}, \ldots, \overline{L^{m}}\right]=d m^{n}$. Положим $p=[k / m]$. Так как $\left(\overline{L^{m}}\right)^{p} \subset \overline{L^{k}}$, то из нижней оценки в $(2)$ получаем $\operatorname{dim}_{\mathbb{C}} \overline{L^{k}} \geqslant \operatorname{dim}_{\mathbb{C}}\left(\overline{L^{m}}\right)^{p} \geqslant \sum_{p-d n^{m}<i \leqslant p} Q(n, i) \approx$ $d m^{n} p^{n} / n ! \approx d k^{n} / n !$. Для доказательства равенства (5) достаточно воспользоваться неравенством (4), так как $F(n, d, k d+1) \approx d k^{n} / n !$.

9. Общий случай. Со всяким пространством $L \in \mathbb{C}(X)$ связано рациональное обобщенное отображсение Веронезе $\rho_{L}: X \rightarrow L^{*}$, определенное формулой $\left\langle\rho_{L}(x), f\right\rangle=f(x)$ для $f \in L$. Обозначим через $X_{L}$ замыкание по Зарисскому образа многообразия $X$ при отображении $\rho_{L}$.

Утверждение 18. Пусть $X$ - неприводимое $n$-мерное алгебраическое многообразие и $L \in \mathcal{K}(X)$. Тогда $[L, \ldots, L]>0$, если и только если $\operatorname{dim} X_{L}=n$.

Доказательство. Если $\operatorname{dim} X_{L}<n$, то почти любое аффинное подпространство в $L^{*}$ коразмерности $n$ не пересекает многообразия $X_{L}$. Поэтому почти любая система уравнений $l_{1}=\cdots=l_{n}=0$, где $l_{i} \in L$, не имеет решений в $X$, т. е. $[L, \ldots, L]=0$. 
Пусть $\operatorname{dim} X_{L}=n$. Положим $X^{*}=X \backslash O$, где $O$ - объединение множества особых точек многообразия $X$ с дивизором полюсов функций из $L$. Образ многообразия $X^{*}$ при обобщенном отображении Веронезе имеет размерность $n$. Поэтому найдется аффинное подпространство коразмерности $n$, трансверсально пересекающее этот образ. Соответствующая этому подпространству система уравнений $l_{1}=\cdots=l_{n}=0$, где $l_{i} \in L$, имеет невырожденные решения в $X^{*}$, поэтому $[L, \ldots, L]>0$.

С отображением $\rho_{L}: X \rightarrow X_{L}$ можно связать алгебраическое многообразие $\widetilde{X}_{L}$, определенное с точностью до бирационального изоморфизма. Перейдем к его определению. Определим поле $\mathbb{C}\left(\widetilde{X}_{L}\right)$ как подполе поля $\mathbb{C}(X)$ рациональных функций на многообразии $X$, постоянных на каждой неприводимой компоненте слоя $\rho^{-1}(z) \subset X$ над каждой точкой $z \in X_{L}$.

Отображение $\rho_{L}^{*}: \mathbb{C}\left(X_{L}\right) \rightarrow \mathbb{C}(X)$ задает вложение поля $\mathbb{C}\left(X_{L}\right)$ в поле $\mathbb{C}\left(\widetilde{X}_{L}\right) \subset \mathbb{C}(X)$. Поле $\mathbb{C}\left(\widetilde{X}_{L}\right)$ является конечным расширением своего подполя $\rho_{L}^{*}\left(\mathbb{C}\left(X_{L}\right)\right)$, так как число неприводимых компонент в слое $\rho^{-1}(z) \subset X$ ограничено общей константой (не зависящей от точки $z \in X_{L}$ ).

Определение 7. Определим $\widetilde{X}_{L}$ как алгебраическое многообразие, поле рациональных функций которого изоморфно полю $\mathbb{C}\left(\widetilde{X}_{L}\right)$.

Вложения $\mathbb{C}\left(\widetilde{X}_{L}\right) \subset \mathbb{C}(X)$ и $\rho_{L}^{*}: \mathbb{C}\left(X_{L}\right) \rightarrow \mathbb{C}\left(\widetilde{X}_{L}\right)$ индуцируют отображения, которые мы будем обозначать через $\widetilde{\pi}_{L}: X \rightarrow \widetilde{X}_{L}$ и $\widetilde{\rho}_{L}: \widetilde{X}_{L} \rightarrow X_{L}$. Из определения видно, что отображение $\widetilde{\rho}_{L}$ имеет конечную степень и что $\operatorname{dim} X_{L}=\operatorname{dim} \widetilde{X}_{L}$. Если отображение $\rho_{L}: X \rightarrow X_{L}$ имеет конечную степень, то многообразия $\widetilde{X}_{L}$ и $X$ бирационально изоморфны.

Утверждение 19. Если функиия $f \in \mathbb{C}(X)$ удовлетворяет алгебраическому уравнению над подполем $\rho_{L}^{*}\left(\mathbb{C}\left(X_{L}\right)\right) \subset \mathbb{C}(X)$, то $f \in \mathbb{C}\left(\widetilde{X}_{L}\right)$.

Доказательство. Если $f^{k}+\rho_{L}^{*}\left(a_{1}\right) f^{k-1}+\cdots+\rho_{L}^{*}\left(a_{k}\right)=0$, где $a_{i} \in \mathbb{C}\left(X_{L}\right)$, то функция $f$ постоянна на каждой неприводимой компоненте прообраза $\rho^{-1}(z) \subset$ $X$ каждой точки $z \in X_{L}$.

Теорема 20. Пусть $X$ - неприводимое алгебраическое многообразие, $L \in$ $\mathcal{K}(X), \operatorname{dim} X_{L}=p, \widetilde{L}=\rho_{L}^{*}(L) \in \mathcal{K}\left(\widetilde{X}_{L}\right) u[\widetilde{L}, \ldots, \widetilde{L}]=d$. Тогда

(i) $d>0$;

(ii) $\bar{H}_{L}(k) \leqslant F(p, d, k d+1)$;

(iii) если $\widetilde{\rho}_{L}: \widetilde{X}_{L} \rightarrow X_{L}-$ бирациональный изоморфизм, то

$$
\sum_{k-d<i \leqslant k} Q(p, i) \leqslant H_{L}(k) \quad u \quad[\widetilde{L}, \ldots, \widetilde{L}]=\lim _{k \rightarrow \infty} \frac{p ! H_{L}(k)}{k^{p}}=\lim _{k \rightarrow \infty} \frac{p ! \bar{H}_{L}(k)}{k^{p}} ;
$$

(iv) для любого $L \in \mathcal{K}(X)$ имеем $[\widetilde{L}, \ldots, \widetilde{L}]=\lim _{k \rightarrow \infty} p ! \bar{H}_{L}(k) / k^{p}$.

Доказательство. В условиях теоремы $H_{L}(k)=\operatorname{dim}_{\mathbb{C}}(\widetilde{L})^{k}$ и $\bar{H}_{L}(k)=$ $\operatorname{dim}_{\mathbb{C}} \overline{\left(\widetilde{L}^{k}\right)}$. Поэтому теорема сводится к вопросу об индексе самопересечения пространства $\widetilde{L}$ на многообразии $\widetilde{X}_{L}$.

(i) Положительность индекса самопересечения $d$ вытекает из равенства размерностей $\operatorname{dim} \widetilde{X}_{L}=\operatorname{dim} X_{L}$ и утверждения 18. 
(ii) вытекает из неравенства (4) в теореме 17, примененной к многообразию $\widetilde{X}_{L}$ и пространству $\widetilde{L}$.

(iii) В условиях п. (iii) пространство $\widetilde{L}$ разделяет общие точки многообразия $\widetilde{X}_{L}$. Поэтому п. (iii) вытекает из теоремы 14. Пункт (iv) вытекает из теоремы 17.

\section{ЛитерАТУРА}

[1] А. Г. Хованский, Многогранник Нъютона, полином Гилъберта и суммъ конечнъх множеств, Функц. анализ и его прил., 26:4 (1992), 57-63.

[2] В. А. Тиморин, А. Г. Хованский, Многогранники и уравнения, в кн.: Математическое просвещение, сер. 3, вып. 14, 2010, 30-57.

[3] А. Г. Хованский, Многогранники Нъютона и торические многообразия, Функц. анализ и его прил., 11:4 (1977), 56-64.

[4] А. Г. Хованский, Многогранники Нъютона и род полных пересечений, Функц. анализ и его прил., 12:1 (1978), 51-61.

[5] K. Kaveh, A. G. Khovanskii, Convex bodies and algebraic equations on affine varieties, http://arxiv.org/abs/0804.4095v1; краткий вариант с названием Algebraic equations and convex bodies, in: Perspectives in Analysis, Topology and Geometry, Progress in Math., Birkhäuser (в печати).

[6] R. Lazarsfeld, M. Mustata, Convex bodies associated to linear series, Ann. Sci. Ec. Norm., 42:5 (2009), 783-835.

[7] K. Kaveh, A. G. Khovanskii, Mixed volume and an extension of intersection theory of divisors, Mosc. Math. J., 10:2 (2010), 343-375.

[8] K. Kaveh, A. G. Khovanskii, Newton-Okounkov convex bodies, semigroups of integral points, graded algebras and intersection theory, http://arxiv.org/abs/0904.3350v1; представлено в Ann. of Math.

[9] K. Kaveh, A. G. Khovanskii, Newton polytopes for horospherical spaces, Mosc. Math. J., 11:2 (2011), 265-283.

[10] K. Kaveh, A. G. Khovanskii, Moment polytopes, semigroup of representations and Kazarnovskii's theorem, J. Fixed Point Theory Appl., 7:2 (2010), 401-417.

[11] K. Kaveh, A. G. Khovanskii, Convex bodies associated to actions of reductive groups, http://arxiv.org/abs/1001.4830; представлено в Mosc. Math. J.

[12] О. Зарисский, П. Самюэль, Коммутативная алгебра, Т. 2, ИЛ, М., 1963, доп. 4.

Институт системного анализа РАН

Независимый московский университет

The University of Toronto, Canada

e-mail: askold@math.toronto.edu
Поступило в редакцию 7 декабря 2010 г. 